\title{
Aortic pressure-diameter relation in patients with non-insulin dependent diabetes mellitus: new insights
}

\author{
K. Toutouzas, C.Stefanadis, E. Tsiamis, Ch. Vlachopoulos, D. Tousoulis, C. Tsioufis, P. Toutouzas \\ Department of Cardiology, University of Athens, Athens, Greece
}

\begin{abstract}
Aims/hypothesis. Type II (non-insulin-dependent) diabetes mellitus is associated with macrovascular disease. Therefore, we investigated the aortic elastic properties by a new method in patients with diabetes and control patients matched with them.

Methods. Patients with Type II diabetes $(n=20)$ and control patients without diabetes $(n=21)$ were enrolled in the study. All patients had coronary artery disease. Instantaneous aortic diameter was measured by an intravascular catheter developed in our institution. Instantaneous aortic pressure was measured simultaneously at the same aortic level with a catheter-tip micromanometer. Thus, aortic pressure-diameter loops were obtained and slope and intercept were calculated. Aortic distensibility, stiffness constant and energy loss were also calculated.

Results. The mean age and the heart rate were similar in the two groups. The pulsatile changes in aortic diameter were greater in the control group $(0.94 \pm 0.4$
\end{abstract}

vs $1.28 \pm 0.4 \mathrm{~mm}, p<0.01)$. The stiffness of the aortic wall was greater in diabetic patients as indicated from the following variables: the distensibility was less in patients with diabetes $\left(1.16 \pm 0.6\right.$ vs $1.95 \pm 0.9 \mathrm{~cm}^{2}$. dyne $\left.^{-1} \cdot 10^{-6}, \quad p<0.01\right)$; the slope was greater $(113.4 \pm 120.1$ vs $51.61 \pm 3.3 \mathrm{mmHg} / \mathrm{mm}, p<0.01)$ and the intercept was less in diabetic patients $(-2301.9 \pm 2692.9$ vs $-1114.45 \pm 295.6 \mathrm{mmHg}, \quad p<$ $0.01)$; the stiffness constant was greater in patients with diabetes $\left(1.66 \pm 1.8\right.$ vs $0.77 \pm 0.8 \mathrm{~mm}^{-1}$, $p<0.03$ ). Aortic energy loss was, however, similar between the groups.

Conclusion/interpretation. In patients with non-insulin dependent diabetes aortic elastic properties, evaluated by pressure-diameter relation, are impaired. This could play an important part in the development of vascular complications related to diabetes. [Diabetologia (2000) 43: 1070-1075]

Keywords Aortic distensibility, non-insulin dependent diabetes mellitus

\section{Introduction}

The most common cause of disability and death among subjects with Type II (non-insulin-dependent) diabetes mellitus is macrovascular disease. Type II diabetes is associated with potentially serious complications and exerts further deleterious effects through functional modifications of the arterial wall. The reasons and mechanisms for macrovascular disease in

\section{Received: 17 April 2000}

Corresponding author: K. Toutouzas MD, 24 Karaoli and Dimitriou str., Holargos 15562, Athens, Greece subjects with Type II diabetes are insufficiently known. It has been suggested that the combined effect of increased glucose, insulin, and triglyceride concentrations has a considerable influence on arterial stiffness and could play a part in the pathophysiology of macrovascular disease in Type II diabetes [1].

Investigation of the function of the arterial wall in living subjects has been difficult. Several authors have measured the pulse-wave velocity in the arterial tree with animal experiments or preparates obtained from cadavers [2, 3]. In addition, other studies found higher pulse-wave velocity in diabetic patients, suggesting lower arterial compliance of the arteries [4-6]. Furthermore, repeated non-invasive estimates 
of arterial stiffness indexes in patients with Type II diabetes have been made [7]. In these studies peripheral arteries, such as common carotid and femoral arteries, were evaluated. Aorta, however, functions not only as a conduit delivering blood to the tissues but also as an important modulator of the entire cardiovascular system, buffering the intermittent pulsatile output from the heart to provide a steady flow to capillary beds. Thus, previous studies do not provide insights into the mechanisms that alter the elastic properties of the aorta. Such information is provided with aortic pressure-diameter relation. We described a new method to obtain aortic pressure-diameter relation in conscious humans [8-11]. With this method, aortic diameters were measured with a high-fidelity intravascular catheter, developed in our institution, that incorporates an ultrasonic displacement meter at its distal end. Aortic pressures were recorded simultaneously at the same aortic level with a catheter-tip micromanometer. The purpose of our study was to investigate, using this high-fidelity method for the determination of pressure-diameter relation, the effect of Type II diabetes on aortic performance in patients with coronary artery disease compared with a control group.

\section{Subjects and methods}

Study cohort. All patients with stable angina or positive treadmill stress test with angiographically documented coronary artery disease were considered eligible for inclusion in the study. Patients with congenital heart disease, hypertrophic cardiomyopathy, a left ventricular ejection fraction less than $55 \%$, chronic obstructive pulmonary disease, congestive heart failure or any other systemic disease were excluded from the protocol. Thereafter, patients with a history of Type II diabetes were examined and only patients without major non-cardiac complications from the diabetes were included. Type II diabetes was defined as fasting glucose more than $7.77 \mathrm{mmol} / \mathrm{l}$ or a history of diabetes or both or being treated with oral hypoglycaemic medication. Patients were excluded if information was missing about antidiabetic medication or if they had received insulin injections. Finally, 20 patients with Type II diabetes fulfilling the above criteria were selected. Moreover, 21 patients matched with them for sex and distribution of risk factors of coronary artery disease were included in the control group. All patients discontinued medication for at least five half-lives before the study, except aspirin and antidiabetic medication. Coronary artery disease of the study cohort was documented by coronary angiography. Fasting glucose, total cholesterol, high density lipoprotein cholesterol and triglyceride concentrations were estimated in all patients. The protocol was approved by the institutional ethics committee and all patients gave informed consent before participation.

Measurement of aortic diameter and pressure. Instantaneous aortic diameter and pressure were recorded simultaneously at the same level of the aorta. The method has been previously described and validated [8-11]. The aortic diameter was measured with a Y-shaped intravascular catheter (Cat.No.5RER060, Cordis, Europa N. V., Roden, The Netherlands). At each

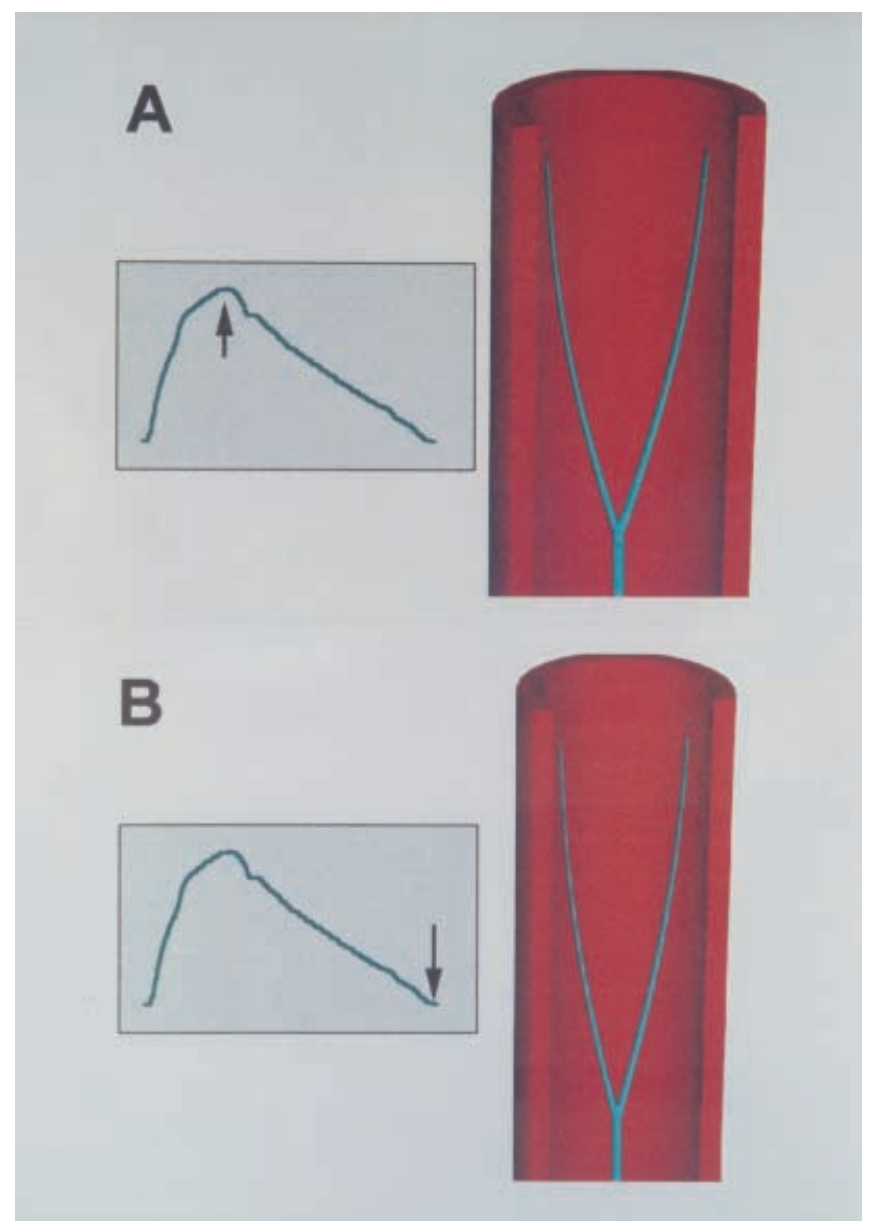

Fig. 1A, B. Schematic representation of the diameter-measuring device, which follows the aortic wall movement during the cardiac cycle. In $\mathbf{A}$, the ultrasonic crystals measure the diameter of the aorta in systole and in $\mathbf{B}$ in diastole

distal arm of the catheter a piezoelectric crystal ( $5 \mathrm{MHz}$ in frequency, 1-mm; Crystal Biotech, Hapkinton, Mass., USA) is attached. These crystals are stabilized on the aortic wall following the movement of the aorta during the cardiac cycle (Fig.1). The resolution of assessing changes in diameter is $10 \mu \mathrm{m}$ with flat frequency response in testing up to $40 \mathrm{MHz}$.

In addition, aortic pressure was recorded with a catheter-tip micromanometer (model SPC-330; Millar Instruments, Houston, Texas, USA). The transducer was calibrated electronically against mercury at the beginning of each study.

Study protocol. All studies were carried out under controlled room temperature of $20 \pm 1{ }^{\circ} \mathrm{C}$. Immediately after standard diagnosis using a catheter, an aortogram was obtained from each patient and those with abnormal lumen irregularities suggesting the presence of atheromatic plaques were excluded. Thereafter, the aortic function was studied. A heparin bolus (100 U/ $\mathrm{kg}$ ) was injected before the introduction of the Y-shaped catheter and the micromanometer and during the procedure the activated clotting time was maintained above $300 \mathrm{~s}$.

An $8 \mathrm{~F} 50-\mathrm{cm}$ in length guiding sheath was introduced through a $9 \mathrm{~F}$ introducer (both from Cordis Europa N. V.) from the right femoral artery and positioned at the level of the proximal descending aorta under fluoroscopic observation. Through this guiding sheath the diameter device, with the wires collapsed, was advanced. Once the catheter was in posi- 
tion the guiding sheath was withdrawn. Thus, the arms of the Y-shaped end of the catheter were spread apart and were stabilized on the aortic wall. Thus, the arms of the catheter could freely follow the movement of the aorta during the cardiac cycle (Fig. 1).

Subsequently, the $3 \mathrm{~F}$ catheter-tip micromanometer was inserted through a $5 \mathrm{~F}$ introductory sheath into the punctured left femoral artery and advanced slightly below the exact level of the pair of crystals.

All measurements were obtained $30 \mathrm{~min}$ after the last infusion of contrast medium. Arterial pressure and ECG were continuously recorded.

Data acquisition. We collected ECG, aortic diameter and aortic pressure signals data with a VF-1 mainframe (Crystal Biotech) fitted with appropriate modules for acquisition of data throughout the study. The recordings were displayed in realtime mode on a PC (Pentium 100) using a multichannel 12-bit analogue-to-digital converter (Data Translation, Marlboro, Mass., USA) and commercially available data acquisition software (Dataflow; Crystal Biotech). All signals were digitized every $5 \mathrm{~ms}$. The digitized data were stored and later processed by commercially available software (Microsoft Excel for Windows). We analysed data on ten consecutive cycles and averaged the results of derivative calculations.

Indexes of aortic elastic properties. On the basis of aortic pressure and diameter measurements the following indexes of aortic elastic properties were calculated:

1. Aortic cross-sectional distensibility defined as: $2 \times$ (systolicdiastolic diameter $) /($ diastolic diameter $\times$ pulse pressure).

2. Aortic pressure-diameter relation obtained from plotting the pressure against the diameter digitized data with commercially available computer software (Microsoft Excel for Windows). To characterize the pressure-diameter relation and determine the aortic loop orientation, the slope and intercept of the linear regression line of pressure against diameter were calculated (Fig. 2).

3. Aortic stiffness constant calculated using aortic pressure-diameter data obtained during the ventricular ejection period, which corresponds to the ascending limb of the loop (Fig. 3) $[9,11]$.

4. Aortic energy loss due to the viscosity of the aortic wall was represented by the area $(\mathrm{mm} \times \mathrm{mmHg})$ within the aortic loop which was calculated by the integral of the aortic loop (Microsoft Excel for Windows).

Statistical analysis. Data are presented as means \pm SD. For data with normal distribution the unpaired $t$ test was used. The Mann-Whitney rank-sum test was used to compare data without normal distribution between the two groups. The relation between two variables was evaluated with a linear regression analysis. Probability values of less than 0.05 were considered statistically significant.

\section{Results}

Age, body surface area and the distribution of other major cardiovascular risk factors (family history, hypertension, current smoking and concentrations of cholesterol) were similar between the groups. Heart rate at the time of measurements was similar. Fasting serum glucose concentration was higher in the Type

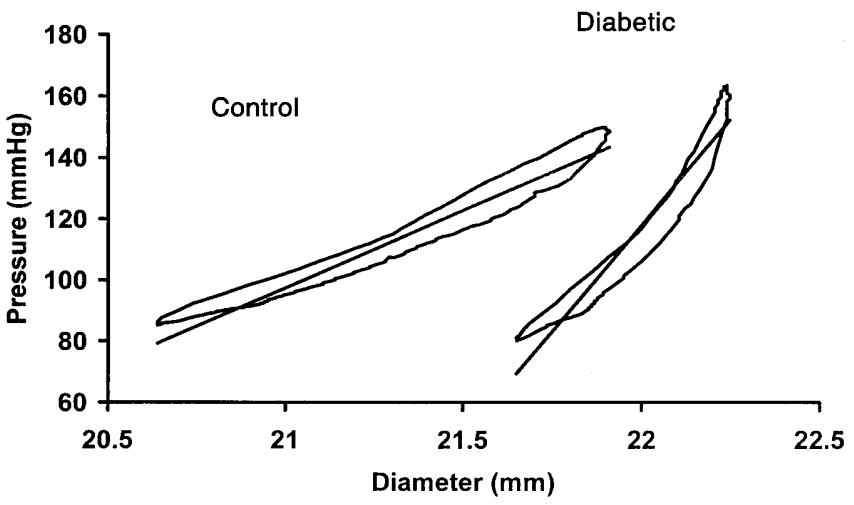

Fig. 2. Representative aortic pressure-diameter loops of a patient with Type II diabetes $\left(\mathrm{y}=137.52 \mathrm{x}-2907.9, r^{2}=0.906\right)$ and a control subject $\left(y=50.416 x-961.42, r^{2}=0.9382\right)$. The loop of the diabetic patient is shifted upward and rightward indicating deterioration of the elastic properties of the aorta. The slope of the linear regression line is greater in the patient with diabetes showing increased aortic stiffness.

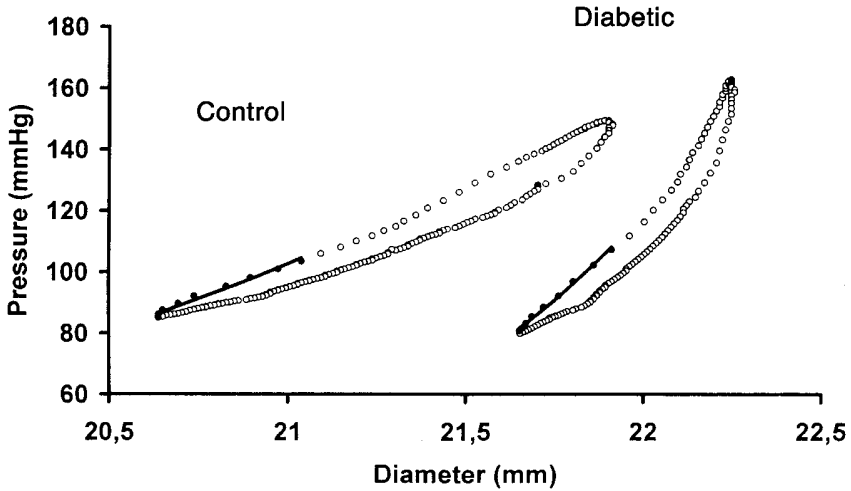

Fig.3. Representative aortic pressure-diameter data used for calculation of the aortic stiffness constant. The stiffness constant expresses the slope of the exponential curve which was fitted to the aortic pressure-diameter data during the left ventricular ejection period. In the diabetic patient $\left(\mathrm{y}=4 \mathrm{E}-09 \mathrm{e}^{1.0944 \mathrm{x}}, r^{2}=0.9954\right)$ the slope is steeper than in the control subject $\left(\mathrm{y}=0.0045 \mathrm{e}^{0.4775 \mathrm{x}}, r^{2}=0.9775\right)$

II diabetes group. Systolic, diastolic and pulse pressures were not different between the two groups. Additionally, the aortic end-diastolic diameters were similar. The pulse diameter was significantly lower in diabetic patients compared with the control group (Table 1).

Aortic function. Distensibility was lower in Type II diabetic patients $(p<0.01)$ (Table 1$)$. The clockwise pressure-diameter loops of Type II diabetic patients were shifted upward and rightward indicating reduced elasticity compared with control subjects (Fig.2). Moreover, a steeper slope and a lower intercept was observed in the group of patients with Type II diabetes $(p<0.01)$. The aortic stiffness constant was greater in these patients $(p=0.03)$ (Fig. 3). Ener- 
Table 1. Characteristics of study cohort and indexes of aortic elastic properties

\begin{tabular}{|c|c|c|c|}
\hline & Control & Type II diabetes & $p$ value \\
\hline$n$ & 21 & 20 & \\
\hline Age (years) & $57.12 \pm 9$ & $59.12 \pm 9$ & NS \\
\hline Body surface area $\left(\mathrm{m}^{2}\right)$ & $1.85 \pm 0.6$ & $1.87 \pm 0.5$ & NS \\
\hline Hypertension & 8 & 7 & NS \\
\hline Current smoking & 7 & 8 & NS \\
\hline Heart rate (beats/min) & $74.12 \pm 14.3$ & $75.42 \pm 11.5$ & NS \\
\hline Fasting glucose $(\mathrm{mmol} / \mathrm{l})$ & $5.28 \pm 0.19$ & $7.08 \pm 0.82$ & $<0.01$ \\
\hline Systolic diameter $(\mathrm{mm})$ & $20.64 \pm 2.9$ & $21.85 \pm 1.5$ & NS \\
\hline Diastolic diameter (mm) & $21.91 \pm 2.1$ & $22.13 \pm 1.6$ & NS \\
\hline Pulse diameter (mm) & $1.28 \pm 0.4$ & $0.94 \pm 0.4$ & $<0.01$ \\
\hline Systolic pressure $(\mathrm{mmHg})$ & $133.53 \pm 17.6$ & $131.5 \pm 19.8$ & NS \\
\hline Diastolic pressure $(\mathrm{mmHg})$ & $72.52 \pm 9.1$ & $75.12 \pm 8.5$ & NS \\
\hline Pulse pressure $(\mathrm{mmHg})$ & $61.34 \pm 6.7$ & $60.2 \pm 5.6$ & NS \\
\hline Mean pressure $(\mathrm{mmHg})$ & $92.34 \pm 10.7$ & $90.5 \pm 9.9$ & NS \\
\hline Distensibility $\left(\mathrm{cm}^{2} \cdot\right.$ dyne $\left.^{-1} \cdot 10^{-6}\right)$ & $1.95 \pm 0.9$ & $1.16 \pm 0.6$ & $<0.01$ \\
\hline Stiffness constant $\left(\mathrm{mm}^{-1}\right)$ & $0.77 \pm 0.8$ & $1.66 \pm 1.8$ & $<0.03$ \\
\hline Slope $(\mathrm{mmHg} / \mathrm{mm})$ & $51.61 \pm 3.3$ & $113.41 \pm 122.2$ & $<0.01$ \\
\hline Intercept $(\mathrm{mmHg})$ & $-1114.45 \pm 295.6$ & $-2301.92 \pm 2692.9$ & $<0.01$ \\
\hline$r^{2}$ & $0.9 \pm 0.07$ & $0.88 \pm 0.07$ & NS \\
\hline Energy loss $(\mathrm{mm} \cdot \mathrm{mmHg})$ & $5.6 \pm 1.3$ & $6.3 \pm 1.4$ & NS \\
\hline
\end{tabular}

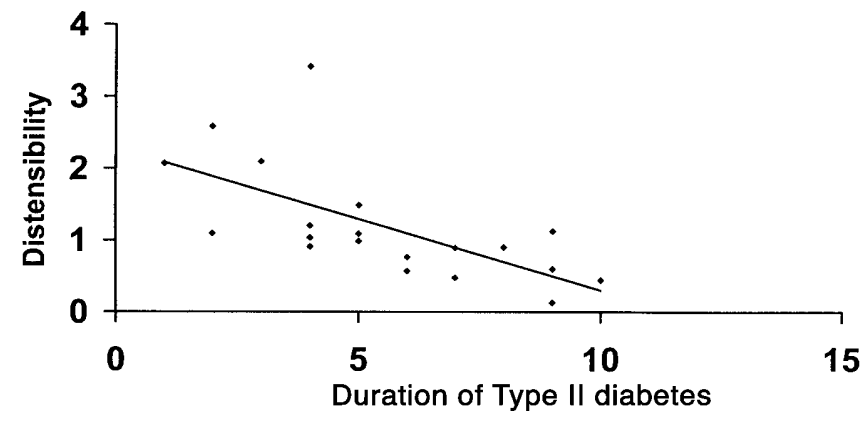

Fig.4. Correlation between the duration of known Type II diabetes (years) and the aortic distensibility $\left(\mathrm{cm}^{2} \cdot\right.$ dyne $\left.\mathrm{e}^{-1} \cdot 10^{-6}\right)$. Linear regression analysis was used to study the association between these variables. $\mathrm{r}=0.65, p<0.001$

gy loss was, however, similar between the two groups $(p=\mathrm{NS})$. An inverse correlation was found between the known duration of Type II diabetes and the aortic distensibility $(r=0.65, p<0.001)$ (Fig. 4).

\section{Discussion}

Our study shows the aortic pressure-diameter relation in diabetic patients using a high-fidelity method. The results indicate that aortic distensibility is reduced in diabetic patients compared with patients without Type II diabetes. Moreover, increased concentration of fasting glucose was associated with decreased aortic distensibility. In addition, the duration of known diabetes correlated with the distensibility.

The method we used provides an accurate determination of pressure-diameter relation. The diameter-measuring device has been previously validated experimentally and clinically [8-11]. In addition, pressure was measured by a catheter-tip micromanometer which allows perfect reproduction of pressure waveforms. The simultaneous recording of pressure and diameter at the same level of the aorta provides reliable calculation of the pressure-diameter relation. This method is unique for the study of aortic mechanics. The distensibility can be accurately calculated. Moreover, the slope of the pressure-diameter relation can be calculated indicating clearly the reduced elastic properties of the aorta in diabetic patients. Additionally, the pressure-diameter loop provides insights into the aortic energetics. Specifically, the area within the loop represents the energy dissipated due to the viscosity of the aortic wall.

The results of this study confirm that elastic properties of the aorta are compromised in the presence of Type II diabetes. Several studies have suggested that diabetes is associated with increased stiffening of the arterial wall [4-7]. Other studies have shown that in normoalbuminuric patients with Type I (insulin-dependent) diabetes mellitus, vascular stiffness is either similar to or greater than that in healthy control subjects [12-13]. In addition, other investigators using non-invasive methods have observed differences in stiffness between femoral and common carotid arteries [12, 14]. One study using echo-tracking sonography has shown that stiffness of aorta and common carotid artery is increased in diabetic women but not in diabetic men [15]. The findings of our study are in agreement with earlier reports of increased arterial stiffness in diabetic patients, without sex-related differences [4-7].

Although distensibility was lower in diabetic patients, the aortic energy loss measured from the pressure-diameter loop was similar between the two groups. Accordingly, the loss of energy due to the vis- 
cosity of the aortic wall is not changed in Type II diabetes.

The underlying mechanisms implicated in the compromise of aortic elastic properties in patients with Type II diabetes, however, are not well known. A disordered collagen metabolism possibly contributes to this unfavourable outcome and also to the same generalized phenomenon affecting both heart and vessels [16]. Moreover, in diabetes an increase in the aortic pepsin-insoluble collagen to total collagen ratio was observed [17]. Furthermore, early glycation could play an important part in structural changes of the arterial wall [18]. Thus, the increased arterial stiffness could be attributed to glycation-induced inter-molecular cross-links [19]. These changes in structural components of the arterial wall could account for stiffening of the aorta. In addition, endothelialcell dysfunction could contribute to alterations in the arterial wall tone. It is well documented that endothelium-dependent relaxation is impaired in diabetic patients [20-22]. These chronic changes in aortic structural components and in endothelial function suggest that alteration of aortic elastic properties are directly related to the duration of Type II diabetes. In this study we found a good correlation between the distensibility and the known duration of diabetes, confirming previous reports [17].

In our study patients with greater fasting glucose concentrations had decreased distensibility. This finding is in agreement with other publications which suggested a strong association between blood glucose concentrations and arterial stiffness [7]. It seems that aortic function is compromised in chronic hyperglycaemia as acute systemic hyperglycaemia is not responsible for changes in arterial distensibility [23].

A limiting factor of the method is its invasive nature. Nevertheless, our method is best suited for reliable measurement of the aortic pressure-diameter relation. Furthermore, the technique is safe because complications were not encountered either in in this or previous studies [8-11].

In our study we attempted to limit the possible effect of other cardiovascular risk factors in aortic distensibility. Thus, we included patients with coronary artery disease in both groups. In addition, all patients were similar in age, smoking habits and their cholesterol concentrations. Furthermore, we excluded patients with aortic lumen irregularities.

The effect of Type II diabetes on aortic function seems to be unfavourable. This observation could have important clinical implications. Recently, our group showed that aortic stiffness is a risk factor for recurrent acute coronary events in patients with ischaemic heart disease [24]. Diabetic patients with coronary artery disease have a poor prognosis even with coronary intervention or medical treatment [25]. Accordingly, treatment with pharmaceutical compounds targetting factors that regulate the aortic function could be considered for diabetic patients. The endothelial function has an important role in the mechanical behaviour of arteries and a recent study indicated that angiotensin-converting enzyme inhibition improves the endothelial function in patients with Type II diabetes [26].

The impaired aortic elastic properties in diabetic patients could increase the afterload for the systolic function of the left ventricle, thus leading to deterioration in the performance of this cardiac cavity resulting in diabetic cardiomyopathy. The exact mechanism whereby diabetic cardiomyopathy develops is uncertain and several mechanisms have been proposed including small and microvascular disease, autonomic dysfunction, metabolic derangements, and interstitial fibrosis [27-28]. The impairment of the aortic function could also be considered as an additional pathophysiologic mechanism for the progression of diabetic cardiomyopathy.

Moreover, future studies with a larger number of patients could correlate the aortic function indexes with the concentration of indicators of glycaemic control, such as glycated haemoglobin. In addition, the exact period of the initiation of the aortic function deterioration in the presence of Type II diabetes needs to be studied as well as the effect on the survival of diabetic patients.

Our study confirms that elastic properties of the aorta are deteriorated in diabetic patients. Moreover, the duration of known Type II diabetes was correlated with the distensibility of the aorta. Thus, Type II diabetes has a considerable effect on aortic elastic properties and could have an important role in the pathophysiologic process of vascular complications related to Type II diabetes.

Acknowledgements. This study was supported by a grant from the Hellenic Heart Foundation.

\section{References}

1. Barrett-Connor E, Orchard T (1984) Diabetes and heart disease. In: Harris M (ed.) Diabetes in America. Washington, D. C. NIH XVI pp 1-41

2. Bramwell JV, Hill AV (1992) Velocity of transmission of the pulse wave velocity and elasticity of arteries. Lancet 1 : 891-892

3. McDonald DA (1968) Regional pulse-wave velocity in the arterial tree. J Appl Physiol 24: 73-78

4. Lo CS, Relf IRN, Myers KA, Wahlquist ML (1986) Doppler recognition of preclinical changes in arterial wall in diabetic subjects and pulse-wave damping. Diabetes Care 9: 27-31

5. Vavuranakis M, Stefanadis C, Triantafillidi E, Toutouzas K, Toutouzas P (1999) Coronary artery distensibility in diabetic patients with simultaneous measurements of luminal area and intracoronary pressure: evidence of impaired reactivity to nitroglycerin. J Am Coll Cardiol 34(4): 1075-1081

6. Neutel JM, Smith DHG, Graettinger WF, Weber MA (1992) Dependency of arterial compliance on circulating 
neuroendocrine and metabolic factors in normal subjects. Am J Cardiol 69: 1340-1344

7. Salomaa V, Riley W, Kark JD, Nardo C, Folsom AR (1995) Non-insulin-dependent diabetes mellitus and fasting glucose and insulin concentrations are associated with arterial stiffness indexes. The ARIC Study. Atherosclerosis Risk in Communities Study. Circulation 91(5): 1432-1443

8. Stefanadis C, Stratos C, Vlachopoulos C et al. (1995) Pressure-diameter relation of the human aorta: a new method of determination by the application of a special ultrasonic dimension catheter. Circulation 92: 2210-2219

9. Stefanadis C, Dernellis J, Vlachopoulos C, et al. (1997) Aortic function in arterial hypertension determined by pressure-diameter relation: effects of diltiazem. Circulation 96: $1853-1858$

10. Stefanadis C, Vlachopoulos C, Tsiamis E et al. (1998) Unfavorable effects of passive smoking on aortic function in men. Ann Intern Med 128: 426-434

11. Stefanadis C, Dernellis J, Vavuranakis M, et al. (1998) Effects of ventricular pacing-tachycardia on aortic mechanics in man. Cardiovasc Res 39: 506-514

12. Kool MJF, Lambert J, Stehouwer CDA, Hoeks APG, Struijker Boudier HAJ, van Bortel LMAB (1995) Vessel wall properties of large arteries in uncomplicated insulindependent diabetes mellitus (IDDM). Diabetes Care 18: 618-624

13. Christensen T, Neubauer B (1987) Arterial wall stiffness in insulin-dependent diabetes mellitus. Acta Radiol 28: 207-209

14. Lambert J, Pijpers R, van Ittersum FJ et al. (1997) Sodium, blood pressure, and arterial distensibility in insulin-dependent diabetes mellitus. Hypertension 30: 1162-1168

15. Ryden Ahlgren A, Lanne T, Wollmer P, Sonesson B, Hasen F, Sundkvist G (1995) Increased arterial stiffness in women, but not in men with IDDM. Diabetologia 38: 1082-1089

16. Monnier VM, Bautista O, Kenny D et al. (1999) Skin collagen glycation, glycoxidation, and crosslinking are lower in subjects with long-term intensive versus conventional therapy of type 1 diabetes: relevance of glycated collagen products versus $\mathrm{HbA} 1 \mathrm{c}$ as markers of diabetic complications. DCCT Skin Collagen Ancillary Study Group. Diabetes Control and Complications Trial. Diabetes 48: 870-880
17. Meng J, Sakata N, Takebayashi S et al. (1996) Advanced glycation end products of the Maillard reaction in aortic pepsin-insoluble and pepsin-soluble collagen from diabetic rats. Diabetes 45: 1037-1043

18. Iino K, Yoshinari M, Yamamoto M et al. (1996) Effect of glycated collagen on proliferation of human smooth muscle cells in vitro. Diabetologia 39: 800-806

19. Sims TJ, Rasmussen LM, Oxlund H, Bailey AJ (1996) The role of glycation cross-links in diabetic vascular stiffening. Diabetologia 39: 946-951

20. Tesfamarian B (1994) Free radicals in diabetic endothelial cell dysfunction. Free Radic Biol Med 16: 391-393

21. Johnstone MT, Creager SJ, Scales KM, Cusco JA, Lee BK, Creager MA (1993) Impaired endothelium-dependent vasodilation in patients with insulin-dependent diabetes mellitus. Circulation 88: 2510-2516

22. Sakamoto S, Minami K, Niwa Yet al. (1998) Effect of exercise training and food restriction on endothelium-dependent relaxation in the otsuka long-evans tokushima rat, a model of spontaneous NIDDM. Diabetes 47: 82-86

23. Lambert J, Smulders RA, Aarsen M, Gallay FP, Stehouwer CD (1997) The acute effect of hyperglycaemia on vessel wall properties. Scand J Clin Lab Invest 57: 409-414

24. Stefanadis C, Dernellis J, Vlachopoulos C et al. (2000) Aortic stiffness as a risk factor for recurrent acute coronary events in patients with ischemic heart disease. Eur Heart $\mathbf{J}$ 21: 390-396

25. Gottlieb S, Boyko V, Harpaz D et al. (1999) Long-term (three-year) prognosis of patients treated with reperfusion or conservatively after acute myocardial infarction. Israeli thrombolytic syrvey group. J Am Coll Cardiol 34: 70-82

26. O'Driscoll G, Green D, Maiorana A, Stanton K, Colreavy F, Taylor R (1999) Improvement in endothelial function by angiotensin-converting enzyme inhibition in non-insulin-dependent diabetes mellitus. J Am Coll Cardiol 33: 1506-1511

27. Dhalla NS, Liu X, Panagia V, Takeda N (1998) Subcellular remodeling and heart dysfunction in chronic diabetes. Cardiovasc Res 40: 239-247

28. Spector KS (1998) Diabetic cardiomyopathy. Clin Cardiol 21: $885-887$ 